\title{
Sharp Estimates for Dirichlet Eigenfunctions in Horn-Shaped Regions
}

\author{
Rodrigo Bañuelos ${ }^{1}$, Burgess Davis ${ }^{2}$ \\ 1 Department of Mathematics, Purdue University, W. Lafayette, IN 47907, USA \\ 2 Department of Statistics, Purdue University, W. Lafayette, IN 47907, USA
}

Received: 13 October 1993

Commun. Math. Phys. 150, 209-215 (1992)

Professors G. Verchota and A. Vogel have pointed out an error in parts (b) and (c) of Theorem 1 in [1]. To correct this, parts (b) and (c) should be replaced by $\left(\mathrm{b}^{\prime}\right)$ and $\left(\mathrm{c}^{\prime}\right)$ below. These changes do not affect the statement or the proof of part (a), which was the main result of the paper. We adopt the notation of [1] and assume throughout that $\theta(x)>0, \theta(x) \downarrow 0$.

(b') Assume $\theta \in L^{1}$. Then for all $x>1$,

$$
\left|\varphi_{\lambda}(x, 0)\right| \leqq C_{\varphi_{\lambda}} e^{-\pi / 2 \int_{1}^{x} \frac{d t}{\theta(t)}}
$$

$\left(c^{\prime}\right)$ Assume $\left|\theta^{\prime}(x)\right| \leqq m$ for all $x$. Then for $x>1$,

$$
\varphi(x, 0) \geqq c_{m} \varphi(1,0) \exp \left(\frac{-\pi}{2} \int_{1}^{x} \frac{d t}{\theta(t)}-\frac{\pi}{24} \int_{1}^{x} \frac{\theta^{\prime}(t)^{2}}{\theta(t)} d t\right) .
$$

For the proof of $\left(\mathrm{c}^{\prime}\right)$ replace Lemma 1.2 of [1] by Theorem 2.1 of Haliste, (reference 7 of [1]), which shows that the harmonic measure $\omega_{(x, 0)}^{D_{1}}\left(I_{1}\right)$ is bounded below by $c_{m}$ times the exponential appearing in (2), and continue exactly as in the proof of (c). We note that $\left(\mathrm{c}^{\prime}\right)$ still proves the sharpness of (a) which was the main reason for (c). Indeed, since $\theta^{\prime}$ is bounded, the Harnack inequality shows that there are constants $c_{1}$ and $c_{2}$ depending on $m$ such that for $z=(x, y), 0<y<c_{1} \theta(x)$, $\varphi(x, y) \geqq c_{2} \varphi(x, 0)$. This and $\left(\mathrm{c}^{\prime}\right)$ with a $\theta$ for which the second factor in the exponential is bounded give examples where the area of $D_{\theta}$ is infinite and the left-hand side of $(0.3)$ of [1] is infinite when $\varepsilon=0$, and where the area of $D_{\theta}$ is finite and the left-hand side of $(0.3)$ is infinite for any $\varepsilon<0$.

For $\left(b^{\prime}\right)$, choose $x_{\lambda}$ large enough and depending only on $\lambda$ such that the lowest eigenvalue $\mu$ of the domain $D(\lambda)=\left\{z \in D_{\theta}: x>x_{\lambda}\right\}$ satisfies $\lambda<\mu$. Let $I_{\lambda}=\{z \in$ $\left.D_{\theta}: x=x_{\lambda}\right\}$. By Theorem 3.2 of reference 7 of [1], it suffices to show that for $x>x_{\lambda}$, $\left|\varphi_{\lambda}(x, 0)\right| \leqq C_{\varphi_{\lambda}} \omega_{(x, 0)}^{D(\lambda)}\left(I_{\lambda}\right)$. If we let $B_{t}$ be Brownian motion in $D(\lambda)$ and 
$\eta$ its exit time from $D(\lambda)$, it follows from the Feynman-Kac formula that $\varphi_{\lambda}(x, 0)=E_{x}\left(e^{\lambda \eta} \varphi_{\lambda}\left(B_{\eta}\right)\right)$. Since $\varphi_{\lambda}$ is zero on the boundary of $D(\lambda)$ except on $I_{\lambda}$, we have after conditioning, that

$$
\left|\varphi_{\lambda}(x, 0)\right| \leqq \sup _{\xi \in I_{\lambda}}\left|\varphi_{\lambda}(\xi)\right| \sup _{\substack{z \in D(\lambda) \\ \xi \in I_{\lambda}}} E_{\bar{z}}^{\xi}\left(e^{\lambda \eta}\right) \omega_{(x, 0)}^{D(\lambda)}\left(I_{\lambda}\right)
$$

where $E_{x}^{\xi}$ denotes expectation with respect to the Brownian motion starting at $z$ and conditioned to exit $D(\lambda)$ at $\xi$. Since $\lambda<\mu$, and the area of $D(\lambda)$ is finite, it follows from Bañuelos and Davis [2], that the second sup in (3) is bounded by a constant depending on $\lambda$ and this proves $\left(b^{\prime}\right)$.

\section{References}

1. Bañuelos, R., Davis, B.: Sharp estimates for Dirichlet Eigenfunctions in horn-shaped regions. Commun. Math. Phys. 150, 209-215 (1992)

2. Bañuelos, R., Davis, B.: Heat kernel, eigenfunctions and conditioned Brownian motion in planar domains. J. Funct. Anal. 89, 188-200 (1989)

Communicated by B. Simon 